\title{
Feasible sets, comparative risk aversion, and comparative uncertainty aversion in bargaining
}

Citation for published version (APA):

Driesen, B. W. I., Lombardi, M., \& Peters, H. J. M. (2015). Feasible sets, comparative risk aversion, and comparative uncertainty aversion in bargaining. Maastricht University, Graduate School of Business and Economics. GSBE Research Memoranda No. 031 https://doi.org/10.26481/umagsb.2015031

Document status and date:

Published: 01/01/2015

DOI:

10.26481/umagsb.2015031

Document Version:

Publisher's PDF, also known as Version of record

\section{Please check the document version of this publication:}

- A submitted manuscript is the version of the article upon submission and before peer-review. There can be important differences between the submitted version and the official published version of record.

People interested in the research are advised to contact the author for the final version of the publication, or visit the DOI to the publisher's website.

- The final author version and the galley proof are versions of the publication after peer review.

- The final published version features the final layout of the paper including the volume, issue and page numbers.

Link to publication

\footnotetext{
General rights rights.

- You may freely distribute the URL identifying the publication in the public portal. please follow below link for the End User Agreement:

www.umlib.nl/taverne-license

Take down policy

If you believe that this document breaches copyright please contact us at:

repository@maastrichtuniversity.nl

providing details and we will investigate your claim.
}

Copyright and moral rights for the publications made accessible in the public portal are retained by the authors and/or other copyright owners and it is a condition of accessing publications that users recognise and abide by the legal requirements associated with these

- Users may download and print one copy of any publication from the public portal for the purpose of private study or research.

- You may not further distribute the material or use it for any profit-making activity or commercial gain

If the publication is distributed under the terms of Article $25 \mathrm{fa}$ of the Dutch Copyright Act, indicated by the "Taverne" license above, 


\section{Maastricht University}

Bram Driesen, Michele Lombardi, Hans Peters

Feasible sets, comparative risk aversion, and comparative uncertainty aversion in bargaining

RM/15/031

\section{GSBE}

Maastricht University School of Business and Economics

Graduate School of Business and Economics

P.O Box 616

NL- 6200 MD Maastricht

The Netherlands 


\title{
Feasible sets, comparative risk aversion, and comparative uncertainty aversion in bargaining
}

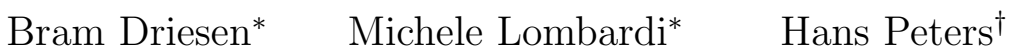

This version, November 2015

\begin{abstract}
We study feasible sets of the bargaining problem under two different assumptions: the players are subjective expected utility maximizers or the players are Choquet expected utility maximizers. For the latter case, we consider the effects on bargaining solutions when players become more risk averse and when they become more uncertainty averse.
\end{abstract}

JEL Classification: C78, D81

Keywords: Subjective expected utility, Choquet expected utility, comparative risk aversion, comparative uncertainty aversion, bargaining

\section{Introduction}

In the classical bargaining problem of Nash (1950) a number of bargainers face the task of finding a unanimous agreement over the expected utility allocations resulting from the lotteries, i.e. finite probability distributions, over a set of alternatives. In this paper we extend the bargaining problem by adopting the Savage (1954) framework of decision making under uncertainty. We study the consequences for the feasible set of a bargaining problem under two different assumptions about the way bargainers make decisions: as subjective expected utility maximizers or as Choquet expected utility maximizers. In the latter case, the preferences of a bargainer are characterized by a utility function for riskless alternatives and lotteries, and a capacity (non-additive probability measure) for uncertain states of the world. Thus, the attitude of a bargainer towards risk can be strictly separated from his attitude towards uncertainty, and we use this feature to derive results about the effects of comparative risk aversion and comparative uncertainty aversion on the outcomes assigned by specific (namely, monotonic) bargaining solutions.

*Adam Smith Business School, University of Glasgow, Glasgow, G12 8QQ, United Kingdom, e-mail: bram.driesen@glasgow.ac.uk, michele.lombardi@glasgow.ac.uk.

${ }^{\dagger}$ Department of Quantitative Economics, Maastricht University, P.O.Box 616, NL-6200 MD Maastricht, Netherlands, e-mail: h.peters@maastrichtuniversity.nl. 
The applications that we have in mind concern bargaining situations where the agreement that the parties reach may be contingent on the future state of the world that will materialize. For instance, the wages and labor conditions in an agreement in the traditional bargaining problem between an employer and a labor union may be made dependent on the future state of the economy.

\subsection{Related literature}

Most closely related to the present work is Köbberling and Peters (2003). In that paper, the bargaining problem is extended by assuming that probabilities in lotteries may be transformed by a probability weighting function, and overall utilities are rank dependent (Quiggin, 1982). The paper distinguishes between (comparative) utility risk aversion as expressed by concavity of the utility function for lotteries and (comparative) probabilistic risk aversion as expressed by convexity of the probability weighting function. The effects of these attitudes on bargaining solutions are in line with what we find in the present paper.

There are several papers, notably Safra and Zilcha (1993), Volij and Winter (2002), and Rubinstein et al (1992), which study the qualitative predictions of the Nash bargaining solution beyond the expected utility framework. Roth and Rothblum $(1982)^{1}$ show that under the Nash bargaining solution, it is disadvantageous to play against a more risk averse opponent, even in the case of risky outcomes, provided that lotteries only attach positive probabilities to alternatives that are preferred over the riskless disagreement alternative. Safra and Zilcha (1993) show that this result breaks down if the expected utility assumption is abandoned. This is confirmed by results in the present paper (see Example 5.7). Volij and Winter (2002) show that increased risk aversion of one bargainer is beneficial for that bargainer and hurts his opponent, given that both bargainers are risk loving (so that the outcome is a lottery) in a model adopting Yaari's (1987) dual theory of choice under risk. On the other hand, Rubinstein et al (1992) find that under their extension of the Nash bargaining solution it is disadvantageous for a bargainer to be more risk averse, a result which is not confirmed in our framework (see again Example 5.7). A prudent conclusion that may be drawn from all these results is that the Nash bargaining solution behaves irregularly under changes in risk attitudes, and that in this respect it is also sensitive to the way in which risk and uncertainty are modelled beyond the expected utility assumption. As will appear again in the present paper (but for instance also in Safra and Zilcha, 1993, or Köbberling and Peters, 2003) bargaining solutions that are monotonic tend to behave much more regularly under changes in attitude towards risk and uncertainty. Examples of such solutions are the Kalai-Smorodinsky (Raiffa, 1953; Kalai and Smorodinsky, 1975) and egalitarian (Kalai, 1977) solutions.

\footnotetext{
${ }^{1}$ For other results on comparative risk aversion in the classical bargaining model under expected utility see Kannai (1977), Kihlstrom et al (1981), Wakker et al (1986), and Safra et al (1990).
} 


\subsection{This paper}

The set of riskless alternatives in this paper is the set of all divisions of one unit of a perfectly divisible good among $n$ bargainers or players. Each player has a strictly increasing and concave utility function which depends only on the own share of the good. Lotteries over riskless alternatives are included as well, and under certainty the utility of a lottery is its expected utility. Further, a finite set of states of the world is assumed.

In Section 3 we follow Savage (1954) and assume that each player attaches subjective utilities to the states of the world. An act assigns to each state of the world a riskless alternative or lottery. We show that the feasible set resulting from considering all possible acts is comprehensive, compact and convex, and strictly comprehensive if each player attaches positive probability to each state of the world.

The results in Section 3 form the basis for those in Section 4, where we assume that the players' probability assessments over the states of the world can be non-additive, and are described by capacities; players are then assumed to be Choquet expected utility maximizers (Schmeidler, 1986, 1989). We show that in this case the feasible set is convex if the capacities are convex. Moreover, we define and characterize increased uncertainty aversion in terms of capacities and increased risk aversion in terms of the utility functions for lotteries.

In Section 5, we show that for a monotonic bargaining solution, increased uncertainty aversion of a player is disadvantageous for the opponents, whereas increased risk aversion is advantageous for the opponents. We also show that a more uncertainty averse player prefers his allocation to the one obtained by his less uncertainty averse alter ego; whereas a less risk averse player prefers his allocation to the one obtained by his more risk averse alter ego. For nonmonotonic bargaining solutions these results may break down, as we show by examples for the Nash bargaining solution.

Section 6 concludes.

\section{Preliminaries}

Following Savage (1954), the analytical framework of decision making under uncertainty involves a set of states of the world, a set of consequences, and a set of acts - i.e., functions that map states of the world to consequences. Resolution of uncertainty reveals the unique true state of the world, and thus, given an act, provides a decision maker with certainty about the realized consequence.

The situation under consideration in this paper is one with multiple decision makers, or players. Specifically, the player set is $N=\{1, \ldots, n\}$ where $n \in \mathbb{N}$, $n>1$. The set of states of the world is $\Omega=\{1, \ldots, K\}$ where $K \in \mathbb{N}, K>1$. The set of consequences is the set of all lotteries - probability distributions with finite support - on the set

$$
A=\left\{a \in \mathbb{R}_{+}^{n} \mid \sum_{i=1}^{n} a_{i} \leq 1\right\}
$$


of all possible divisions of one unit of a perfectly divisible good among the $n$ players. ${ }^{2}$ An act is a map $f$ assigning an element of $A$ or lottery over $A$ to every state of the world, and $F$ is the set of all acts.

For each player $i$, a function $U_{i}$, mapping acts into the reals, represents $i$ 's preferences if for all $f, g \in F$, we have $U_{i}(f) \geq U_{i}(g)$ if and only if $i$ prefers act $f$ to $g$. In this paper we consider different specifications of the functions $U_{i}$, and for these specifications examine the properties of the set

$$
S=\left\{\left(U_{1}(f), \ldots, U_{n}(f)\right) \mid f \in F\right\} .
$$

For the sake of brevity, we write $U(f) \equiv\left(U_{1}(f), \ldots, U_{n}(f)\right)$ for every $f \in F$.

\section{Subjective Expected Utility}

In this section, the players in $N$ are assumed to be Subjective Expected Utility (SEU) maximizers (Savage, 1954). The premise of the SEU model is that each player $i$ evaluates an act $f \in F$ based on his subjective belief about the states of the world. Specifically, player $i$ 's evaluation of an act $f \in F$ is given by

$$
U_{i}(f)=\sum_{k \in \Omega} \pi_{i}(k) u_{i}\left(f_{i}(k)\right),
$$

where $\pi_{i}: \Omega \rightarrow[0,1]$ represents his subjective probability distribution over the state space $\Omega$, and the continuous, strictly increasing, and concave function $u_{i}:[0,1] \rightarrow \mathbb{R}$ is player $i$ 's expected utility function for lotteries: if $f(k)$ is a lottery then with some abuse of notation $u_{i}\left(f_{i}(k)\right)$ denotes player $i$ 's expected utility for the lottery $f_{i}(k)$ resulting from $f(k)$. Since the representation of a player's preference is unique up to positive linear transformations, we may assume without loss of generality that $u_{i}(0)=0$ and $u_{i}(1)=1$ for every $i \in N$.

For the sake of brevity, we denote $u(a)=\left(u_{1}\left(a_{1}\right), \ldots, u_{n}\left(a_{n}\right)\right)$ for every $a \in A$. The set $\hat{S}=\{u(a) \mid a \in A\}$ contains all possible utility evaluations attainable in any one state of the world $k \in \Omega{ }^{3}$ Since $\hat{S}$ does not depend on the realized state of the world, it is immediate that $S=\hat{S}$ whenever players' beliefs about the realization of those states coincide. More generally, $\hat{S}$ is a subset of $S$. To see this, observe that for any $u(a) \in \hat{S}$, the act $f$ with $f(k)=a$ for all states $k$ satisfies $U(f)=u(a)$.

The set $\hat{S}$ satisfies a number of appealing properties: it is non-empty, compact, convex, and strictly comprehensive. ${ }^{4}$ We omit the obvious proof of this

\footnotetext{
${ }^{2}$ Here, $\mathbb{R}_{+}^{n}=\left\{x \in \mathbb{R}^{n} \mid x \geq 0\right\}$. We use the following vector inequalities: for all $x, y \in \mathbb{R}^{n}$, $x \geq y$ if $x_{i} \geq y_{i}$ for each $i, x>y$ if $x \geq y$ and $x \neq y$, and $x \gg y$ if $x_{i}>y_{i}$ for each $i$.

${ }^{3}$ Due to concavity of the utility functions we do not need to include lotteries in the definition of $\hat{S}$. In fact, in this paper lotteries merely play an (only) implicit motivational role later on when we define the concept of comparative risk aversion. See also Section 6 about generalizing the set of consequences.

${ }^{4} \mathrm{~A}$ set $T \subseteq \mathbb{R}^{n}$ is said to be comprehensive if $x \in T$ and $0 \leq y \leq x$ together imply $y \in T$. The set $T$ is strictly comprehensive if in addition, $y \neq x$ implies the existence of $z \in T$ with $z \gg y$.
} 
but, in what follows, we verify which of these properties carry over when uncertainty is introduced.

Proposition 3.1 Under SEU, the set $S$ is comprehensive.

Proof. Let $x \in S$, and let $y \in \mathbb{R}^{n}$ be such that $0 \leq y \leq x$. It is sufficient to show that $y \in S$.

By definition of $S$, there is an $f \in F$ such that $U(f)=x$. Since each $u_{i}$ is strictly increasing, the inverse function $v_{i}$ exists, so $v_{i}\left(u_{i}\left(a_{i}\right)\right)=u_{i}\left(v_{i}\left(a_{i}\right)\right)=$ $a_{i}$ for all $a \in A$. Consider the function $g: \Omega \rightarrow \mathbb{R}^{n}$ defined by $g(k)=$ $\left(v_{1}\left(z_{1}(k)\right), \ldots, v_{n}\left(z_{n}(k)\right)\right)$ where

$$
z_{i}(k)= \begin{cases}\frac{y_{i}}{x_{i}} \cdot u_{i}\left(f_{i}(k)\right) & \text { if } x_{i}>0 \\ 0 & \text { otherwise }\end{cases}
$$

for each $i \in N$ and $k \in \Omega$. Since $0 \leq z_{i}(k) \leq u_{i}\left(f_{i}(k)\right)$ for all $i$ and $k$, and each $v_{i}$ is strictly increasing, it follows that $v_{i}(0) \leq v_{i}\left(z_{i}(k)\right) \leq v_{i}\left(u_{i}\left(f_{i}(k)\right)\right)$ for all $i$ and $k$. Hence $0 \leq g(k) \leq f(k)$ for all $k \in \Omega$, which by comprehensiveness of $A$ implies that $g(k) \in A$ for each $k \in \Omega$. Hence, $g \in F$, and thus by the definition of $S, U(g) \in S$. Observe that for all $i \in N$,

$$
\begin{aligned}
U_{i}(g) & =\sum_{k \in \Omega} \pi_{i}(k) u_{i}\left(g_{i}(k)\right)=\sum_{k \in \Omega} \pi_{i}(k) z_{i}(k) \\
& =\sum_{k \in \Omega} \pi_{i}(k) \frac{y_{i} u_{i}\left(f_{i}(k)\right)}{U_{i}(f)}=y_{i} \cdot \frac{U_{i}(f)}{U_{i}(f)}=y_{i} .
\end{aligned}
$$

Therefore, $y=U(g)$, so that $y \in S$.

The set $S$ is not necessarily strictly comprehensive. For instance, suppose that $n=K=2$. Assume that each player $i$ has a linear utility function $u_{i}$ on the interval [0,1], and consider subjective beliefs $\pi_{1}$ and $\pi_{2}$ with $\pi_{1}(1)=1$ and $\pi_{2}(1)=\pi_{2}(2)=0.5$. Then the set $S$ is equal to the convex polytope in $\mathbb{R}^{2}$ with vertices at $(1,0),(1,0.5),(0,1)$ and $(0,0)$, which is not strictly comprehensive.

The following proposition says that the set $S$ is strictly comprehensive if the subjective probability distribution of each player has full support.

Proposition 3.2 Under SEU, let $\pi_{i}(k)>0$ for each $i \in N$ and $k \in \Omega$. Then $S$ is strictly comprehensive.

Proof. Let $x \in S$ and $y \in \mathbb{R}_{+}^{n}$ with $x>y$. Then by Proposition 3.1, $y \in S$. The aim is to show that there exists a $z \in S$ with $z \gg y$. Let $i \in N$ with $x_{i}>y_{i}$.

By definition of $S$, there are acts $f$ and $g$ such that $U(f)=x$ and $U(g)=y$. Since $U_{i}(f)>U_{i}(g)$, there must exist a $k^{*} \in \Omega$ such that $f_{i}\left(k^{*}\right)>g_{i}\left(k^{*}\right)$. Take some $0<\varepsilon<f_{i}\left(k^{*}\right)-g_{i}\left(k^{*}\right)$, and define $a \in \mathbb{R}^{n}$ with

$$
a_{j}= \begin{cases}f_{j}\left(k^{*}\right)-\varepsilon & \text { if } j=i \\ f_{j}\left(k^{*}\right)+\frac{1}{n-1} \cdot \varepsilon & \text { if } j \neq i\end{cases}
$$


for all $j \in N$. Since $a_{j} \geq 0$ for all $j \in N$ and $\sum_{j \in N} a_{j}=\sum_{j \in N} f_{j}\left(k^{*}\right) \leq 1$, it follows that $a \in A$. Note that by construction, $a \gg g\left(k^{*}\right)$, and thus, $u(a) \gg$ $u\left(g\left(k^{*}\right)\right)$. Now construct the act $h$ as follows:

$$
h(k)= \begin{cases}a & \text { if } k=k^{*} \\ g(k) & \text { otherwise. }\end{cases}
$$

For all $j \in N$ we have

$$
U_{j}(h)=\sum_{k \in \Omega \backslash\left\{k^{*}\right\}} \pi_{j}(k) u_{j}\left(g_{j}(k)\right)+\pi_{j}\left(k^{*}\right) u_{j}\left(a_{j}\right)>\sum_{k \in \Omega} \pi_{j}(k) u_{j}\left(g_{j}(k)\right)=U_{j}(g),
$$

where the inequality follows from the full support assumption. Hence, for $z=$ $U(h)$ we have $z \in S$ and $z \gg y$, as desired.

Proposition 3.3 Under $S E U$, the set $S$ is compact and convex.

Proof. Since $S$ is the image of the compact set $F$ under the continuous function $U$, it is compact.

To show that $S$ is convex, let $x, y \in S$ and $\lambda \in[0,1]$. We show that $\lambda x+$ $(1-\lambda) y \in S$. By definition, there are acts $f$ and $g$ such that $x=U(f)$ and $y=U(g)$. Observe that for each $k, \lambda f(k)+(1-\lambda) g(k)$ is an element of $C$, and thus, $\lambda f+(1-\lambda) g \in F$. Hence, $U(\lambda f+(1-\lambda) g) \in S$. Since each $u_{i}$ is concave, it follows that $u_{i}\left(\lambda f_{i}(k)+(1-\lambda) g_{i}(k)\right) \geq \lambda u_{i}\left(f_{i}(k)\right)+(1-\lambda) u_{i}\left(g_{i}(k)\right)$ for each $k \in \Omega$. Hence for each player $i, U_{i}(\lambda f+(1-\lambda) g) \geq \lambda U_{i}(f)+(1-\lambda) U_{i}(g)$, so that $0 \leq \lambda x+(1-\lambda) y \leq U(\lambda f+(1-\lambda) g)$. Since $S$ is comprehensive by Proposition 3.1, we conclude that $\lambda x+(1-\lambda) y \in S$, as desired.

It was pointed out earlier that $S$ contains $\hat{S}$, and that $S=\hat{S}$ whenever players agree on a single subjective probability distribution $\pi$ over the state space. It turns out that the converse is true as well: if $S=\hat{S}$, then players' subjective beliefs coincide. For any $T \subseteq \mathbb{R}^{n}$, the Pareto optimal set $P(T)$ is defined by $P(T)=\{t \in T \mid$ there exists no $v \in T$ with $v>t\}$.

Proposition 3.4 Under $S E U, \hat{S}=S$ if and only if $\pi_{i}(k)=\pi_{j}(k)$ for all $i, j \in$ $N$ and all $k \in \Omega$.

Proof. We still have to show the only-if part. Suppose, for contradiction, that (say) $\pi_{1}\left(k^{*}\right)=p>q=\pi_{2}\left(k^{*}\right)$ for some $k^{*} \in \Omega$. Let $z$ be a utility allocation in the relative interior of the Pareto set of $\hat{S}$. By the supporting hyperplane theorem there is a hyperplane

$$
H=\left\{y \in \mathbb{R}^{n} \mid \sum_{j=1}^{n} \lambda_{j} y_{j}=c\right\}
$$

that supports $\hat{S}$ at the point $z$. By strict comprehensiveness of $\hat{S}, \lambda_{j}>0$ for all $j=1, \ldots, n$. Define the function $\psi:[0,1] \rightarrow[0,1]$ by $\psi(\alpha)=\max \{\beta \mid$ 
$\left.\left(\alpha, \beta, z_{3}, \ldots, z_{n}\right) \in \hat{S}\right\}$. Note that $\psi$ is well-defined since $\hat{S}$ is a compact set. Let $x_{1}=z_{1}+\varepsilon$ for some $\varepsilon>0$, and $x_{2}=\psi\left(z_{1}+\varepsilon\right)$. Consider an act $f$ with $u(f(k))=z$ for all $k \in \Omega \backslash\left\{k^{*}\right\}$, and $u\left(f\left(k^{*}\right)\right)=\left(x_{1}, x_{2}, z_{3}, \ldots, z_{n}\right) \in \hat{S}$. Then

$$
\begin{aligned}
\sum_{j=1}^{n} \lambda_{j} U_{j}(f) & =p \lambda_{1} x_{1}+(1-p) \lambda_{1} z_{1}+q \lambda_{2} x_{2}+(1-q) \lambda_{2} z_{2}+\sum_{j=3}^{n} \lambda_{j} z_{j} \\
& =c+p \lambda_{1}\left(x_{1}-z_{1}\right)+q \lambda_{2}\left(x_{2}-z_{2}\right) .
\end{aligned}
$$

If $q=0$, then $\sum_{j=1}^{n} \lambda_{j} U_{j}(f)=c+p \lambda_{1}\left(x_{1}-z_{1}\right)>c$, so that $U(f) \notin \hat{S}$, a contradiction. Now assume $q>0$. Then $\sum_{j=1}^{n} \lambda_{j} U_{j}(f)=c+p \lambda_{1} \varepsilon+\lambda_{2} q\left(\psi\left(z_{1}+\right.\right.$ $\left.\varepsilon)-\psi\left(z_{1}\right)\right)$. Hence, $\sum_{j=1}^{n} \lambda_{j} U_{j}(f) \geq c$ if and only if

$$
\frac{\psi\left(z_{1}+\varepsilon\right)-\psi\left(z_{1}\right)}{\varepsilon} \geq-\frac{\lambda_{1} p}{\lambda_{2} q} \text {. }
$$

Since $\hat{S}$ is convex and strictly comprehensive, $\psi$ is strictly decreasing and concave. Therefore, $\psi$ is differentiable from the right at the point $z_{1}$. In particular, $\lim _{h \downarrow 0}\left(\psi\left(z_{1}+h\right)-\psi\left(z_{1}\right)\right) / h=-\lambda_{1} / \lambda_{2}$. Since $p>q$ it follows that for $\varepsilon$ small enough, $\psi\left(z_{1}+\varepsilon\right)-\psi\left(z_{1}\right) / \varepsilon>-\lambda_{1} p / \lambda_{2} q$, which implies $\sum_{j=1}^{n} \lambda_{j} U_{j}(f)>c$, and thus again the contradiction $U(f) \notin \hat{S}$. This concludes the proof.

The results of this section form the basis for those in the next one, where we consider Choquet expected utility.

\section{Non-Additive Probabilities}

Motivated by criticisms on the Savage framework (e.g., Ellsberg, 1961), Schmeidler (1989) introduced a generalization of Subjective Expected Utility, which assumes that the probabilities agents attach to the occurrence of events need not be additive. At the basis of this model is the concept of a capacity.

Definition 4.1 A capacity is a function $\mu: 2^{\Omega} \rightarrow[0,1]$ with the following properties:

(i) $\mu(\emptyset)=0$ and $\mu(\Omega)=1$,

(ii) for all $E, E^{\prime} \in 2^{\Omega}, E \subseteq E^{\prime}$ implies $\mu(E) \leq \mu\left(E^{\prime}\right)$.

Rather than by a subjective probability measure over the state space, it is now assumed that each player $i$ 's beliefs are represented by a capacity $\mu_{i}$. Acts are evaluated by means of the Choquet integral.

Definition 4.2 For all vectors $x=\left(x_{1}, \ldots, x_{K}\right) \in \mathbb{R}_{+}^{\Omega}$, a player $i$ endowed with capacity $\mu_{i}$ is a Choquet Expected Utility (CEU) maximizer if player $i$ 's evaluation of $x$ is

$$
C_{\mu_{i}}(x)=\sum_{k=1}^{K}\left(x_{\tau(k)}-x_{\tau(k-1)}\right) \mu_{i}(\{\tau(k), \ldots, \tau(K)\}),
$$


where $\tau$ is a permutation of $\Omega$ such that $x_{\tau(1)} \leq x_{\tau(2)} \leq \ldots \leq x_{\tau(K)}$ and $x_{\tau(0)}=0$.

Player $i$ endowed with capacity $\mu_{i}$ and utility $u_{i}$ is a CEU maximizer ${ }^{5}$ if his evaluation of the act $f$ is

$$
U_{i}\left(f ; u_{i}, \mu_{i}\right)=C_{\mu_{i}}(x) \text { where } x=\left(u_{i}\left(f_{i}(k)\right)\right)_{k \in \Omega} .
$$

As before, we are interested in the properties of the set $S=\left\{\left(U_{i}\left(f ; u_{i}, \mu_{i}\right)\right)_{i \in N} \mid\right.$ $f \in F\}$. Note that if a player's capacity is additive, he is an SEU maximizer. Therefore, the results in the previous section are a special case of those obtained here.

Definition 4.3 For all $f, g \in F$ and $i \in N$ with utility function $u_{i}, f$ and $g$ are comonotonic for $i$ if there are no states $k, l \in \Omega$ such that $u_{i}\left(f_{i}(k)\right)>u_{i}\left(f_{i}(l)\right)$ and $u_{i}\left(g_{i}(k)\right)<u_{i}\left(g_{i}(l)\right)$. The set of all comonotonic acts for player $i$ is called a comoncone for $i$.

Each player $i$ evaluates an act $f$ as $\sum_{k \in \Omega} w_{k} u_{i}\left(f_{i}(k)\right)$ where each $w_{k}$ is of the form $\left[\mu_{i}(E \cup\{k\})-\mu_{i}(E)\right]$ with $E \in 2^{\Omega}$, and $\sum_{k \in \Omega} w_{k}=\mu_{i}(\Omega)-\mu_{i}(\emptyset)=1$. It is easily seen from the definition that the weights used in the evaluation of two comonotonic acts, coincide. Thus, for all acts in the same comoncone the weights assigned to different states remain unchanged. Since $w \geq 0$ and $\sum_{k} w_{k}=1$, these weights - for all intents and purposes - may be interpreted as a player's subjective probabilities. This means that our results on $F$ under the assumption of SEU, also hold for the intersections of players' comoncones in $F$ under the assumption of non-additive beliefs. ${ }^{6}$

Let $u=\left(u_{1}, \ldots, u_{n}\right)$ and $\mu=\left(\mu_{1}, \ldots, \mu_{n}\right)$ and let $g \in F$. Let $T=\{U(f ; u, \mu) \mid$ $f$ is comonotonic with $g$ for every $i \in N\}$, where $U(f ; u, \mu)=\left(U_{i}\left(f ; u_{i}, \mu_{i}\right)\right)_{i \in N}$. Then, since any constant act is comonotonic with $g$ for every $i \in N$, the set $T$ trivially contains $\hat{S}$. Furthermore, by the same arguments as in Proposition 3.1 and Proposition 3.3 it follows that $T$ is comprehensive, compact and convex. Also strict comprehensiveness can be obtained by the following condition on players' capacities.

Definition 4.4 A capacity $\mu$ is strictly monotonic if for all $E, E^{\prime} \in 2^{\Omega}, E \varsubsetneqq E^{\prime}$ implies $\mu(E)<\mu\left(E^{\prime}\right)$.

If a player's capacity is strictly monotonic, then he attaches a strictly positive weight $w_{k}>0$ to each state $k \in \Omega$. Hence, if all players' capacities are strictly monotonic, then by the same argument as in Proposition 3.2, we have that $T$ is strictly comprehensive.

\footnotetext{
${ }^{5}$ See Siniscalchi (2008) for a gentle introduction to Choquet expected utility.

${ }^{6}$ This would also follow from Schmeidler's (1986) result that $C_{\mu}$ is first-degree homogeneous, and satisfies comonotonic additivity, i.e. $C_{\mu}(x+y)=C_{\mu}(x)+C_{\mu}(y)$ for any two comonotonic acts $x, y$.
} 
Since $F$ is the union of finitely many (namely, less than $\left.(K !)^{n}\right)$ intersections of players' comoncones, it follows that $S$ is the union of finitely many compact, convex, and comprehensive sets. In addition, if all capacities are strictly monotonic, then the comprehensiveness is strict. Since compactness, comprehensiveness and strict comprehensiveness, are all preserved under finite union, the following obtains as a corollary from the results in the previous section.

Corollary 4.5 Under CEU, the set $S$ is compact and comprehensive. Furthermore, it is strictly comprehensive if players' capacities are strictly monotonic.

Convexity is not preserved under finite union. Indeed, the set $S$ need not be convex, as the following example shows.

Example 4.6 Let $n=K=2$, and let the players have linear utilities. Assume that $\left(\mu_{1}(1), \mu_{1}(2)\right)=\left(\mu_{2}(1), \mu_{2}(2)\right)=(0.9,0.5)$. Then $S=S_{1} \cup S_{2}$, where

$$
S_{1}=\operatorname{conv}\{(0,0),(1,0),(0,1),(0.9,0.5)\}
$$

and

$$
S_{2}=\operatorname{conv}\{(0,0),(1,0),(0,1),(0.5,0.9)\} .
$$

Nontrivial convex combinations of $(0.9,0.5)$ and $(0.5,0.9)$ are not contained in $S .^{7}$

However, it turns out that convexity of players' capacities is a sufficient condition for convexity of $S$.

Definition 4.7 A capacity $\mu$ is convex if $\mu(E)+\mu\left(E^{\prime}\right) \leq \mu\left(E \cup E^{\prime}\right)+\mu\left(E \cap E^{\prime}\right)$ for all $E, E^{\prime} \in 2^{\Omega}$.

Definition 4.8 The core of a capacity $\mu$ is the set

$$
\operatorname{core}(\mu)=\left\{\pi \in \mathbb{R}_{+}^{K} \mid \pi(\Omega)=1 \text { and } \pi(E) \geq \mu(E) \text { for all } E \subseteq \Omega\right\} .
$$

Thus, the core of a capacity consists of all probability measures dominating the capacity values. It is well-known that the core of a convex capacity is nonempty. Schmeidler (1986) shows the following result.

Lemma 4.9 The following statements are equivalent.

(i) The capacity $\mu$ is convex.

(ii) For all $\left(x_{1}, \ldots, x_{K}\right) \in \mathbb{R}^{K}, C_{\mu}(x)=\min _{\pi \in \operatorname{core}(\mu)}\left(\sum_{k \in \Omega} \pi(k) x_{k}\right)$.

Schmeidler subsequently showed that, for a convex capacity $\mu, C_{\mu}(x+y) \geq$ $C_{\mu}(x)+C_{\mu}(y)$ for any two $x, y \in \mathbb{R}^{K}$. A similar argument is used in the proof of the following proposition.

\footnotetext{
${ }^{7}$ Note that this example also shows that Proposition 3.4 does not generalize to the nonadditive probability framework, i.e. if players share the same capacity, this does not necessarily mean that $S$ and $\hat{S}$ coincide.
} 
Proposition 4.10 Assume CEU and suppose that $\mu_{i}$ is convex for each $i \in N$. Then the set $S$ is convex.

Proof. Let $x, y \in S$ and $\lambda \in[0,1]$. We show $\lambda x+(1-\lambda) y \in S$. By definition, there are acts $f$ and $g$ such that $x=U(f ; u, \mu)$ and $y=U(g ; u, \mu)$. Since $\lambda f(k)+(1-\lambda) g(k) \in A$ for each $k \in \Omega$, we have $h=\lambda f+(1-\lambda) g \in F$, hence $U(h ; u, \mu) \in S$. By Corollary 4.5, it is then sufficient to show that

$$
0 \leq \lambda U(f ; u, \mu)+(1-\lambda) U(g ; u, \mu) \leq U(h ; u, \mu) .
$$

Since $x, y \in S \subseteq \mathbb{R}_{+}^{n}$, the first inequality is trivially satisfied. To establish the second inequality, consider any player $i$. Since the capacity $\mu_{i}$ is convex, it follows from Lemma 4.9 that there is a $\pi \in \operatorname{core}\left(\mu_{i}\right)$ such that $U_{i}\left(h ; u_{i}, \pi_{i}\right)=$ $\sum_{k \in \Omega} \pi_{i}(k) u_{i}\left(h_{i}(k)\right)$. Then

$$
\begin{aligned}
U_{i}\left(h ; u_{i}, \pi_{i}\right) & =\sum_{k \in \Omega} \pi_{i}(k) u_{i}\left(\lambda f_{i}(k)+(1-\lambda) g_{i}(k)\right) \\
& \geq \lambda \sum_{k \in \Omega} \pi_{i}(k) u_{i}\left(f_{i}(k)\right)+(1-\lambda) \sum_{k \in \Omega} \pi_{i}(k) u_{i}\left(g_{i}(k)\right) \\
& \geq \lambda U_{i}\left(f ; u_{i}, \pi_{i}\right)+(1-\lambda) U_{i}\left(g ; u_{i}, \pi_{i}\right),
\end{aligned}
$$

where (3) follows from concavity of $u_{i}$ and (4) again from Lemma 4.9. Now (2) follows.

Convexity of players' capacities is a sufficient condition for convexity of $S$, but it is not a necessary condition, as is demonstrated by the following example.

Example 4.11 Let $n=K=2$, and let the players have linear utilities. Assume that $\left(\mu_{1}(1), \mu_{1}(2)\right)=\left(\mu_{2}(2), \mu_{2}(1)\right)=(3 / 4,3 / 5)$. Since $3 / 4+3 / 5>1$, both players' capacities violate convexity. However, $S=\operatorname{conv}\{(0,0),(1,0),(0,1)$, $(3 / 4,3 / 4)\}$, which is convex.

The remainder of this section is devoted to the concepts of comparative uncertainty and risk aversion and the consequences for the feasible set $S$.

Schmeidler (1989) introduces a concept of uncertainty aversion in the CEU framework and shows that it is equivalent to convexity of the capacity. In our model we are interested in comparative uncertainty aversion.

Definition 4.12 Suppose that player $i$ 's preferences over $A$ are represented by the utility function $u_{i}$. (Player $i$ endowed with) capacity $\mu_{i}^{\prime}$ is more uncertainty averse than (player $i$ endowed with) capacity $\mu_{i}$ if

$$
U_{i}\left(f ; u_{i}, \mu_{i}^{\prime}\right) \geq u_{i}(\eta) \Rightarrow U_{i}\left(f ; u_{i}, \mu_{i}\right) \geq u_{i}(\eta) \text { for all } f \text { in } F \text { and all } \eta \in[0,1] .
$$

The interpretation of this definition is that if a more uncertainty averse player (or decision maker) prefers an (uncertain) act over a sure payoff then so does the less uncertainty averse player. Note that in Definition 4.12 we could just as well write ' $\eta$ ' instead of ' $u_{i}(\eta)$ ', but the present definition shows more clearly 
the contrast with the definition of comparative risk aversion (see Definition 4.14 below). ${ }^{8}$

Proposition 4.13 Let $i \in N$ and let $\mu_{i}$ and $\mu_{i}^{\prime}$ be convex capacities. The following statements are equivalent.

(i) For all $E \in 2^{\Omega}, \mu_{i}^{\prime}(E) \leq \mu_{i}(E)$.

(ii) $\operatorname{core}\left(\mu_{i}\right) \subseteq \operatorname{core}\left(\mu_{i}^{\prime}\right)$.

(iii) $\mu_{i}^{\prime}$ is more uncertainty averse than $\mu_{i}$.

Proof. The implication $(i) \Rightarrow(i i)$ is immediate. Now assume (ii); we show (iii). For any $f \in F$, (ii) and Lemma 4.9 imply

$$
U_{i}\left(f ; u_{i}, \mu_{i}^{\prime}\right)=\min _{\pi \in \operatorname{core}\left(\mu_{i}^{\prime}\right)} \sum_{k \in \Omega} \pi(k) x_{k} \leq \min _{\pi \in \operatorname{core}\left(\mu_{i}\right)} \sum_{k \in \Omega} \pi(k) x_{k}=U_{i}\left(f ; u_{i}, \mu_{i}\right)
$$

where $x_{k}=u_{i}\left(f_{i}(k)\right)$ for all $k \in \Omega$. Therefore, for every $\eta \in[0,1], U_{i}\left(f ; u_{i}, \mu_{i}^{\prime}\right) \geq$ $u_{i}(\eta)$ implies $U_{i}\left(f ; u_{i}, \mu_{i}\right) \geq u_{i}(\eta)$, so that (iii) holds.

Finally, assume (iii); we show (i). Let $E \in 2^{\Omega}$, and consider an act $f$ with $f_{i}(k)=\alpha$ if $k \in E$ and $f_{i}(k)=\beta$ if $k \notin E$, where $\alpha$ and $\beta$ are such that $u_{i}(\alpha)>$ $u_{i}(\beta)$. By (iii), it follows that for every $\eta \in[0,1]$ with $U_{i}\left(f ; u_{i}, \mu_{i}^{\prime}\right) \geq u_{i}(\eta)$, we have $U_{i}\left(f ; u_{i}, \mu_{i}\right) \geq u_{i}(\eta)$. By choosing $\eta$ such that $u_{i}(\eta)=U_{i}\left(f ; u_{i}, \mu_{i}^{\prime}\right)$, this implies $U_{i}\left(f ; u_{i}, \mu_{i}\right) \geq U_{i}\left(f ; u_{i}, \mu_{i}^{\prime}\right)$, or equivalently,

$$
\mu_{i}(E) u_{i}(\alpha)+\left(1-\mu_{i}(E)\right) u_{i}(\beta) \geq \mu_{i}^{\prime}(E) u_{i}(\alpha)+\left(1-\mu_{i}^{\prime}(E)\right) u_{i}(\beta) .
$$

Since $u_{i}(\alpha)-u_{i}(\beta)>0$, this implies $\mu_{i}^{\prime}(E) \leq \mu_{i}(E)$, so that (i) follows.

In the present framework, the definition of comparative risk aversion is as follows.

Definition 4.14 Suppose that player $i$ is endowed with capacity $\mu_{i}$. (Player $i$ endowed with) utility function $u_{i}^{\prime}$ is more risk averse than (player $i$ endowed with) utility function $u_{i}$ if

$$
U_{i}\left(f ; u_{i}^{\prime}, \mu_{i}\right) \geq u_{i}^{\prime}(\eta) \Rightarrow U_{i}\left(f ; u_{i}, \mu_{i}\right) \geq u_{i}(\eta) \text { for all } f \in F \text { and } \eta \in[0,1] .
$$

Comparative risk aversion can be characterized as follows.

Proposition 4.15 Suppose that player $i$ is endowed with capacity $\mu_{i}$. The following two statements are equivalent.

(i) $u_{i}^{\prime}$ is more risk averse than $u_{i}$.

(ii) There exists a continuous, strictly increasing and concave function $v$ : $[0,1] \rightarrow[0,1]$ such that $u_{i}^{\prime}=v \circ u_{i}$.

\footnotetext{
${ }^{8}$ In fact, ' $u_{i}(\eta)$ for all $\eta \in[0,1]$ ' in Definition 4.12 may also be replaced by ' $u_{i}(\ell)$ for all lotteries $\ell$ over $[0,1]$ '.
} 
Proof. The implication (i) $\Rightarrow$ (ii) follows from Yaari (1969) by considering constant acts. Now assume that (ii) holds and let $f \in F$ and $\eta \in[0,1]$ such that $U_{i}\left(f ; u_{i}^{\prime}, \mu_{i}\right) \geq u_{i}^{\prime}(\eta)$, i.e., $\sum_{k=1}^{K} \pi_{k} v\left(u_{i}\left(f_{i}(k)\right)\right) \geq v\left(u_{i}(\eta)\right)$ for some probabilities $\pi_{k}, k=1, \ldots, K$. Then by concavity of $v$,

$$
v\left(U_{i}\left(f ; u_{i}, \mu_{i}\right)\right)=v\left(\sum_{k=1}^{K} \pi_{k} u_{i}\left(f_{i}(k)\right)\right) \geq \sum_{k=1}^{K} \pi_{k} v\left(u_{i}\left(f_{i}(k)\right)\right) \geq v\left(u_{i}(\eta)\right) .
$$

Since $v$ is strictly increasing, this implies $U_{i}\left(f ; u_{i}, \mu_{i}\right) \geq u_{i}(\eta)$. Thus, (i) follows.

\section{$5 \quad$ Bargaining}

Assuming that players are CEU maximizers and with notations as in the preceding sections, the set $S$ can be regarded as the feasible set of a bargaining problem with uncertainty. In this context we also write

$$
B(u, \mu)=\{U(f ; u, \mu) \mid f \in F\}=\left\{\left(U_{1}\left(f ; u_{1}, \mu_{1}\right), \ldots, U_{n}\left(f ; u_{n}, \mu_{n}\right)\right) \mid f \in F\right\}
$$

instead of simply $S$. Throughout, capacities are assumed to be convex, so that by Proposition 4.10 the set $B(u, \mu)$ is convex. We adopt the 'welfaristic approach', which means that a bargaining solution depends exclusively on the feasible set; in other words, if $B(u, \mu)=B\left(u^{\prime}, \mu^{\prime}\right)$ for some utility-capacity profiles $(u, \mu)$ and $\left(u^{\prime}, \mu^{\prime}\right)$, then a bargaining solution assigns the same point to both bargaining problems. Formally, let

$$
\mathcal{B}=\left\{S \subseteq \mathbb{R}^{n} \mid S=B(u, \mu) \text { for some } u=\left(u_{i}\right)_{i \in N}, \mu=\left(\mu_{i}\right)_{i \in N}\right\} .
$$

A bargaining solution is a map $\varphi: \mathcal{B} \rightarrow \mathbb{R}^{n}$ such that $\varphi(S) \in S$ for every $S \in \mathcal{B}$. In this section we collect some observations about the effects of increased uncertainty or risk aversion on bargaining solutions.

A possibly plausible condition on a bargaining solution is the following.

Definition 5.1 A bargaining solution $\varphi$ is monotonic if $\varphi(S) \geq \varphi\left(S^{\prime}\right)$ for all $S, S^{\prime} \in \mathcal{B}$ with $S^{\prime} \subseteq S$.

Well-known bargaining solutions satisfying this condition are the KalaiSmorodinsky solution (Kalai and Smorodinsky, 1975) and the proportional bargaining solutions (Kalai, 1977). We make the following observations.

Proposition 5.2 Let $\varphi$ be a monotonic bargaining solution, and let $B(u, \mu) \in$ $\mathcal{B}$.

(i) Let $i \in N$ and $B\left(u, \mu^{\prime}\right) \in \mathcal{B}$ such that $\mu_{j}^{\prime}=\mu_{j}$ for all $j \in N \backslash\{i\}$ whereas $\mu_{i}^{\prime}$ is more uncertainty averse than $\mu_{i}$. Then $\varphi_{j}\left(B\left(u, \mu^{\prime}\right)\right) \leq \varphi_{j}(B(u, \mu))$ for all $j \in N \backslash\{i\}$. 
(ii) Let $i \in N$ and $B\left(u^{\prime}, \mu\right) \in \mathcal{B}$ such that $u_{j}^{\prime}=u_{j}$ for all $j \in N \backslash\{i\}$ whereas $u_{i}^{\prime}$ is more risk averse than $u_{i}$. Then $\varphi_{j}\left(B\left(u^{\prime}, \mu\right)\right) \geq \varphi_{j}(B(u, \mu))$ for all $j \in N \backslash\{i\}$.

Proof. For (i), by Proposition 4.13 and Definition 4.2, we have $B\left(u, \mu^{\prime}\right) \subseteq$ $B(u, \mu)$. For (ii), by Proposition 4.15 (which implies $u_{i}^{\prime}(\eta) \geq u_{i}(\eta)$ for all $\eta \in$ $[0,1])$ and Definition 4.2 , we have $B(u, \mu) \subseteq B\left(u^{\prime}, \mu\right)$. The proposition now follows from monotonicity of $\varphi$.

Proposition 5.2 implies that if a player becomes more uncertainty averse then this is disadvantageous for the other players, but if a player becomes more risk averse then this is advantageous for his opponents. Note that from the proof of the proposition it follows that also the more uncertainty (risk) averse player has lower (higher) Choquet expected utility, but such comparisons are meaningless since we are dealing with different players. However, for the player who is replaced by a more uncertainty averse or more risk averse person we have the observations in Proposition 5.4 below.

Definition 5.3 A bargaining solution $\varphi$ is weakly Pareto optimal if for all $S \in \mathcal{B}$ and $x \in S, x \ngtr \varphi(S)$.

Proposition 5.4 Let $\varphi$ be a monotonic and weakly Pareto optimal bargaining solution, and let $B(u, \mu) \in \mathcal{B}$ such that $\mu_{j}$ is strictly monotonic for every $j \in N$.

(i) Let $i \in N$ and $B\left(u, \mu^{\prime}\right) \in \mathcal{B}$ such that $\mu_{j}^{\prime}=\mu_{j}$ for all $j \in N \backslash\{i\}, \mu_{i}^{\prime}$ is strictly monotonic, and $\mu_{i}^{\prime}$ is more uncertainty averse than $\mu_{i}$. Let $f, f^{\prime} \in F$ satisfy $U(f ; u, \mu)=\varphi(B(u, \mu))$ and $U\left(f^{\prime} ; u, \mu^{\prime}\right)=\varphi\left(B\left(u, \mu^{\prime}\right)\right)$. Then $U_{i}\left(f^{\prime} ; u_{i}, \mu_{i}^{\prime}\right) \geq U_{i}\left(f ; u_{i}, \mu_{i}^{\prime}\right)$.

(ii) Let $i \in N$ and $B\left(u^{\prime}, \mu\right) \in \mathcal{B}$ such that $u_{j}^{\prime}=u_{j}$ for all $j \in N \backslash\{i\}$ and $u_{i}^{\prime}$ is more risk averse than $u_{i}$. Let $f, f^{\prime} \in F$ satisfy $U(f ; u, \mu)=\varphi(B(u, \mu))$ and $U\left(f^{\prime} ; u^{\prime}, \mu\right)=\varphi\left(B\left(u^{\prime}, \mu\right)\right)$. Then $U_{i}\left(f ; u_{i}, \mu_{i}\right) \geq U_{i}\left(f^{\prime} ; u_{i}, \mu_{i}\right)$.

Proof. We prove (i), the proof of (ii) is analogous. By Proposition 5.2 we have $U_{j}\left(f^{\prime} ; u_{j}, \mu_{j}^{\prime}\right) \leq U_{j}\left(f ; u_{j}, \mu_{j}\right)=U_{j}\left(f ; u_{j}, \mu_{j}^{\prime}\right)$ for all $j \in N \backslash\{i\}$. By weak Pareto optimality of $\varphi$ and strict comprehensiveness of $B\left(u, \mu^{\prime}\right)$ this implies $U_{i}\left(f^{\prime} ; u_{i}, \mu_{i}^{\prime}\right) \geq U_{i}\left(f ; u_{i}, \mu_{i}^{\prime}\right)$.

Proposition 5.4 says that a more uncertainty averse player prefers any act giving rise to the bargaining solution outcome in his bargaining problem to any act giving rise to the bargaining solution outcome in the problem with his less uncertainty averse substitute. In contrast, a less risk averse player prefers any act giving rise to the bargaining solution outcome in his bargaining problem to any act giving rise to the bargaining solution outcome in the problem for his more risk averse substitute - of course, under the conditions in the proposition. Combining both propositions, and under the conditions in these propositions, we may say that a player who becomes more uncertainty averse hurts his opponents but does not prefer any act obtained in the solution to the original bargaining 


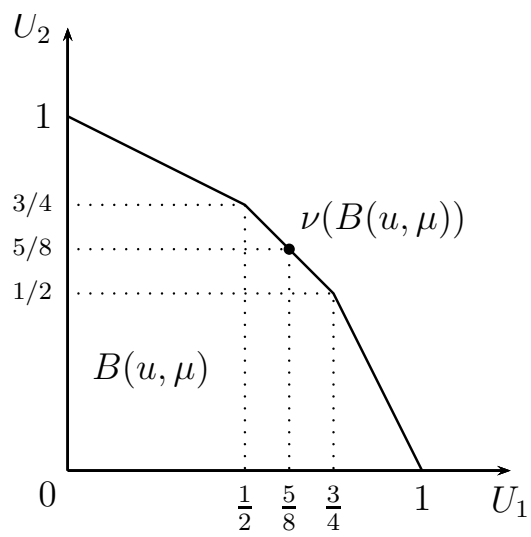

(a) $B(u, \mu)$

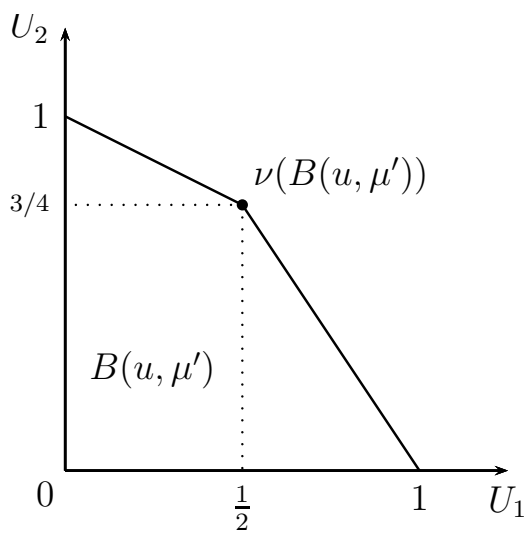

(b) $B\left(u, \mu^{\prime}\right)$

Figure 1: A visual illustration of Example 5.5

problem; and a player who becomes less risk averse hurts his opponents but does not prefer any act obtained in the solution to the original bargaining problem.

The following examples show that Propositions 5.2 and 5.4 do not hold for a non-monotonic bargaining solution such as the Nash bargaining solution $\nu$ (Nash, 1950), which assigns to every $S \in \mathcal{B}$ the point $\nu(S)$ which maximizes the product $\Pi_{i \in N} x_{i}$ over the set $S$.

Example 5.5 Let $N=\{1,2\}$ and $\Omega=\{1,2,3\}$. The players' capacities are $\mu_{1}$ and $\mu_{2}$, with $\mu_{1}(1)=\frac{1}{4}, \mu_{1}(23)=\frac{3}{4}$, and $\mu_{1}(E)=\frac{1}{2}$ for all events $E \notin\{1,23, \Omega\}$; and $\mu_{2}(3)=\frac{1}{4}, \mu_{1}(12)=\frac{3}{4}$, and $\mu_{1}(E)=\frac{1}{2}$ for all events $E \notin\{3,12, \Omega\}$. Let $u_{1}(\alpha)=u_{2}(\alpha)=\alpha$ for every $0 \leq \alpha \leq 1$. Then one can check that

$$
B(u, \mu)=\operatorname{conv}\left\{(0,0),(0,1),\left(\frac{1}{2}, \frac{3}{4}\right),\left(\frac{3}{4}, \frac{1}{2}\right),(1,0)\right\},
$$

and $\nu(B(u, \mu))=\left(\frac{5}{8}, \frac{5}{8}\right)$. This point corresponds uniquely to the act $f=$ $\left((0,1),\left(\frac{1}{2}, \frac{1}{2}\right),(1,0)\right)$. Now let $\mu^{\prime}=\left(\mu_{1}^{\prime}, \mu_{2}\right)$ with $\mu_{1}^{\prime}(23)=\frac{5}{8}$ and $\mu_{1}^{\prime}(E)=\mu_{1}(E)$ otherwise. Then $\mu^{\prime}$ still consists of convex and strictly monotonic capacities, and by Proposition $4.13, \mu_{1}^{\prime}$ is more uncertainty averse than $\mu_{1}$. We now have

$$
B\left(u, \mu^{\prime}\right)=\operatorname{conv}\left\{(0,0),(0,1),\left(\frac{1}{2}, \frac{3}{4}\right),(1,0)\right\},
$$

and $\nu\left(B\left(u, \mu^{\prime}\right)\right)=\left(\frac{1}{2}, \frac{3}{4}\right)$, corresponding to the act $f^{\prime}=((0,1),(0,1),(1,0))$.

It follows that $\frac{3}{4}=\nu_{2}\left(B\left(u, \mu^{\prime}\right)\right)>\nu_{2}(B(u, \mu))=\frac{5}{8}$, so that part (i) of Proposition 5.2 does not hold for $\nu$. Also, $U_{1}\left(f ; u_{1}, \mu_{1}^{\prime}\right)=\frac{9}{16}>\frac{1}{2}=U_{1}\left(f^{\prime} ; u_{1}, \mu_{1}^{\prime}\right)$, so that part (i) of Proposition 5.4 does not hold for $\nu$. 


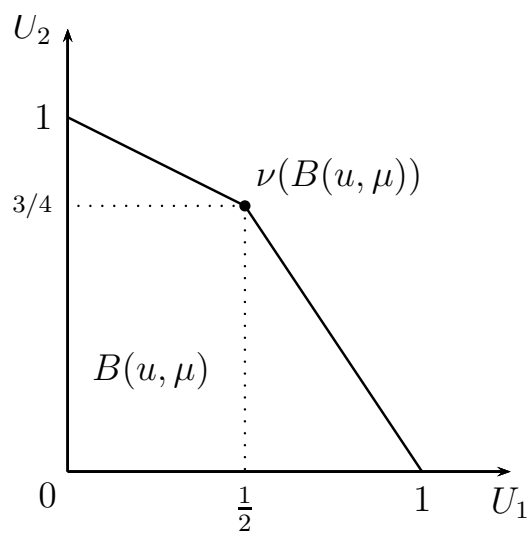

(a) $B(u, \mu)$

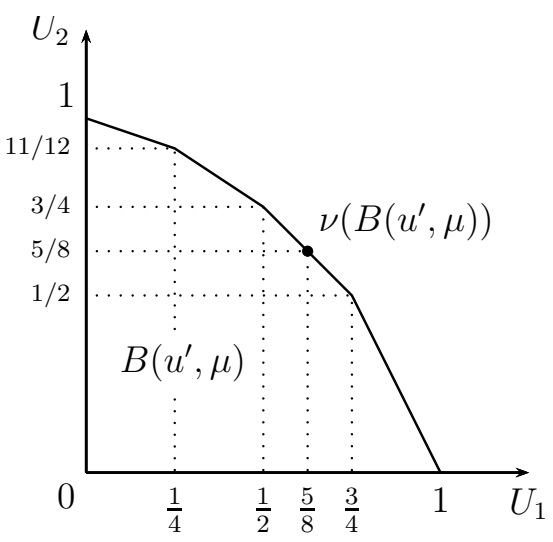

(b) $B\left(u^{\prime}, \mu\right)$

Figure 2: A visual illustration of Example 5.7

Remark 5.6 Example 5.5 shows that parts (i) of Propositions 5.2 and 5.4 do not hold for the Nash bargaining solution. With linear utility functions and convex and strictly monotonic capacities, we need three states for this, which can be shown as follows. Suppose that $\Omega=\{1,2\}$. Then a bargaining problem

$$
B(u, \mu)=\operatorname{conv}\{(0,0),(0,1),(a, b),(c, d),(1,0)\}
$$

with $a+b>1$ and $c+d>1$ can only be realized if either

$$
\left(\mu_{1}(1), \mu_{2}(2)\right)=(a, b) \text { and }\left(\mu_{1}(2), \mu_{2}(1)\right)=(c, d)
$$

or

$$
\left(\mu_{1}(1), \mu_{2}(2)\right)=(c, d) \text { and }\left(\mu_{1}(2), \mu_{2}(1)\right)=(a, b) .
$$

But then $\left[\mu_{1}(1)+\mu_{1}(2)\right]+\left[\mu_{2}(1)+\mu_{2}(2)\right]=(a+c)+(b+d)>2$, which implies that at least one of the two capacities is not convex.

Example 5.7 Let $N=\{1,2\}$ and $\Omega=\{1,2\}$. The players have additive capacities: $\mu_{1}(1)=\mu_{1}(2)=\frac{1}{2}, \mu_{2}(1)=\frac{1}{4}$, and $\mu_{1}(2)=\frac{3}{4}$. Let $u_{1}(\alpha)=u_{2}(\alpha)=\alpha$ for every $0 \leq \alpha \leq 1$. Then

$$
B(u, \mu)=\operatorname{conv}\left\{(0,0),(0,1),\left(\frac{1}{2}, \frac{3}{4}\right),(1,0)\right\},
$$

and $\nu(B(u, \mu))=\left(\frac{1}{2}, \frac{3}{4}\right)$. This point corresponds uniquely to the act $f=$ $((1,0),(0,1))$. Now let $u_{1}^{\prime}:[0,1] \rightarrow \mathbb{R}$ be defined by $u_{1}^{\prime}(\alpha)=\frac{3}{2} \alpha$ if $0 \leq \alpha \leq \frac{1}{3}$ and $u_{1}^{\prime}(\alpha)=\frac{1}{4}+\frac{3}{4} \alpha$ if $\frac{1}{3}<\alpha \leq 1$. Let $u^{\prime}=\left(u_{1}^{\prime}, u_{2}\right)$, then $u_{1}^{\prime}$ is more risk averse than $u_{1}$ by Proposition 4.15, 


$$
B\left(u^{\prime}, \mu\right)=\operatorname{conv}\left\{(0,0),(0,1),\left(\frac{1}{4}, \frac{11}{12}\right),\left(\frac{1}{2}, \frac{3}{4}\right),\left(\frac{3}{4}, \frac{1}{2}\right),(1,0)\right\},
$$

and $\nu\left(B\left(u^{\prime}, \mu\right)\right)=\left(\frac{5}{8}, \frac{5}{8}\right)$. This point corresponds to the act $f^{\prime}=\left((1,0),\left(\frac{1}{6}, \frac{5}{6}\right)\right)$. Now $\nu_{2}\left(B\left(u^{\prime}, \mu\right)\right)=\frac{5}{8}<\frac{3}{4}=\nu_{2}(B(u, \mu))$, so that part (ii) of Proposition 5.2 does not hold for $\nu$. Also, $U_{1}\left(f ; u_{1}, \mu_{1}\right)=\frac{1}{2}<\frac{7}{12}=U_{1}\left(f^{\prime} ; u_{1}, \mu_{1}\right)$, so that part (ii) of Proposition 5.4 does not hold for $\nu$.

\section{Concluding remarks}

In this paper, we have studied properties of the feasible set of a bargaining problem under the assumption that the bargainers are subjective expected utility (SEU) maximizers as well as under the assumption that they are Choquet expected utility (CEU) maximizers. For the latter case, we studied the comparative statics properties of monotonic bargaining solutions with respect to the changes in the degree of risk/uncertainty aversion. We found that an increase in one's opponent's risk aversion is advantageous, and that an increase in one's opponent's uncertainty aversion is disadvantageous. Further, a less risk averse bargainer prefers his allocation to the one obtained by his more risk averse alter ego, whereas a more uncertainty averse bargainer prefers his allocation to the one obtained by his less uncertainty averse alter ego. Finally, we showed by means of examples that the effect of changes in the degree of risk/uncertainty aversion on the Nash bargaining solution is not conclusive.

Since CEU generalizes SEU, it follows that the comparative risk aversion results for monotonic bargaining solutions hold for the SEU case as well. Of course, uncertainty does not play a role in the SEU model.

Our (bargaining) results are derived under the so-called welfaristic assumption: The bargaining solution depends only on the feasible set and not directly on the underlying model. Hence, these results may or may not hold if the welfaristic assumption is dropped.

As to the set of consequences in the paper, which is equal to all divisions of one unit of a perfectly divisible good, this may with some modifications be generalized to an arbitrary compact set of (riskless) alternatives, as in Köbberling and Peters (2003). Our choice in this paper is motivated by convenience, in particular when deriving results about comprehensiveness of feasible sets. Also, if no risk is involved (that is, only uncertainty regarding the state of the world but no lotteries), the results still go through if risk aversion is interpreted as diminishing marginal utility (see Peters, 1992).

\section{References}

Ellsberg D (1961) Risk, ambiguity, and Savage axioms. Quarterly Journal of Economics 75:643-669

Kalai E (1977) Proportional solutions to bargaining situations: interpersonal utility comparisons. Econometrica 45:1623-1630 
Kalai E, Smorodinsky M (1975) Other solutions to Nash's bargaining problem. Econometrica 43:513-518

Kannai Y (1977) Concavifiability and constructions of concave utility functions. Journal of Mathematical Economics 4: 1-56

Kihlstrom RE, Roth AE, Schmeidler D (1981) Risk aversion and solutions to Nash's bargaining problem. In: Moeschlin O, Pallaschke D (eds): Game Theory and Mathematical Economics. North Holland, Amsterdam

Köbberling V, Peters H (2003) The effect of decision weights in bargaining problems. Journal of Economic Theory 110: 154-175

Nash JF (1950) The bargaining problem. Econometrica 18:155-162

Peters H (1992) A criterion for comparing strength of preference, with an application to bargaining. Operations Research 40:1018-1022

Quiggin J (1982) A theory of anticipated utility. Journal of Economic Behavior and Organization 3:324-344

Raiffa H (1953) Arbitration schemes for generalized two-person games. Annals of Mathematics Studies 28:361-387

Roth AE, Rothblum UG (1982) Risk aversion and Nash's solution for bargaining games with risky outcomes. Econometrica 50:639-647

Rubinstein A, Safra Z, Thomson W (1992) On the interpretation of the Nash bargaining solution and its extension to non-expected utility preferences. Econometrica 60:1171-1186

Safra S, Zilcha I (1993) Bargaining solutions without the expected utility hypothesis. Games and Economic Behavior 5:288-306

Safra Z, Zhou L, Zilcha I (1990) Risk aversion in the Nash bargaining problem with risky outcomes and risky disagreement points. Econometrica 58: 961-965

Savage LJ (1954) Foundations of statistics. Wiley, New York

Schmeidler D (1986) Integral representation without additivity. Proceedings of the American Mathematical Society 97:255-261

Schmeidler D (1989) Subjective probability and expected utility without additivity. Econometrica 57:571-587

Siniscalchi M (2008) Ambiguity and ambiguity aversion. In: The New Palgrave Dictionary of Economics, Second Edition, Eds. Steven N. Durlauf and Lawrence E. Blume. Palgrave Macmillan

Volij O, Winter E (2002) On risk aversion and bargaining outcomes. Games and Economic Behavior 41:120-140 
Wakker P, Peters H, van Riel T (1986) Comparisons of risk aversion, with an application to bargaining. Methods of Operations Research 54:307-320

Yaari ME (1969) Some remarks on measures of risk aversion and on their uses. Journal of Economic Theory 1:315-329

Yaari ME (1987) The dual theory of choice under risk. Econometrica 55:95-115 